\section{Visual perceptions induced by intravitreous injections of therapeutic agents}

\begin{abstract}
Purpose The purpose of this study was to conduct a questionnaire-based survey of subjective visual perceptions induced by intravitreous (IVT) injections of therapeutic agents.
\end{abstract}

Patients and methods Patients undergoing an IVT injection of ranibizumab, pegaptanib sodium, or triamcinolone acetonide were administered a questionnaire in the immediate post-injection period and at 2 weeks of follow-up.

${ }^{1}$ Institute of Eye Surgery and Institute of Vision Research, Whitfield Clinic, Butlerstown North, Waterford, Ireland

${ }^{2}$ Macular Pigment Research Group, Waterford Institute of Technology, Waterford, Ireland

Correspondence: $S$ Charalampidou, Institute of Eye Surgery and Institute of Vision Research, Whitfield Clinic, Cork Road, Waterford, Ireland Tel: + 35387241 1425;

Fax: + 35351319849 .

E-mail:sonia.sofia1@ gmail.com

Received: 23 August 2010 Accepted in revised form: 22 November 2010 Published online: 28 January 2011

This study was presented at the 10th Euretina Congress on the 3 September 2010, in Paris, France and at the Irish College of Ophthalmologists' Annual Conference on the 30 April 2010, in Dublin, Ireland.
Results In the immediate post-injection period (75 IVT injections, 75 eyes, 75 patients), lights and floaters were reported after 20 (27\%) and $24(32 \%)$ IVT injections, respectively. In comparison, at the 2-week follow-up, the incidence of reported lights $(11 ; 15 \%)$ was similar $(P>0.05)$, but the incidence of reported floaters was higher $(48 ; 64 \% ; P=0.00)$.

Subgroup analysis for various injection subgroups (no previous injection vs previous injection(s) in the study eye; injections in study eyes with good VA (logarithm of minimal angle of resolution $[\log M A R] \leq 0.3$ ) vs moderate VA $(0.7<\log M A R>0.3)$ vs poor VA ( $\log M A R \geq 0.7)$; injections according to pharmacological agent (ranibizumab $v s$ pegaptanib vs triamcinolone acetonide); injections in study eyes with choroidal neovascularization (of various causes) vs study eyes with macular edema (of various causes); and injections in phakic $v s$ pseudophakic eyes) did not reveal any statistically significant associations. Visual perceptions experienced following 15\% of IVT injections gave cause for concern to the patient (mean visual analog scale score ( $\pm S D): 4.5( \pm 1.7)$ ), and in $64 \%$ of cases, the patients believed that preoperative counseling would have averted the concern. Conclusions Lights and floaters are frequent visual perceptions following IVT injections of
S Charalampidou', J Nolan², GO Ormonde' and S Beatty ${ }^{1,2}$

therapeutic agents. They can give rise to concern that could be alleviated with preinjection counseling.

Eye (2011) 25, 494-501; doi:10.1038/eye.2010.229; published online 28 January 2011

Keywords: visual experience; intravitreous injection; anti-vascular endothelial growth factor; triamcinolone; visual perception; questionnaire

\section{Introduction}

Intravitreous (IVT) administration of therapeutic agents has become routine practice in ophthalmology in the last decade. IVT injections of antibodies to vascular endothelial growth factor (anti-VEGF), such as ranibizumab (Lucentis, Genentech, San Francisco, CA, USA), pegaptanib sodium (Macugen, OSI/Eyetech and Pfizer, New York, NY, USA) and bevacizumab (Avastin, Genentech), as well as IVT injections of triamcinolone acetonide (Kenalog, Bristol-Myers Squibb, New York, NY, USA), have gained popularity in the treatment of a wide array of ophthalmic disorders, such as choroidal neovascularization (CNV) secondary to neovascular age-related macular degeneration ${ }^{1,2}$ and angioid streaks, ${ }^{3}$ macular edema (MO) secondary to diabetes mellitus, ${ }^{4,5}$ central retinal vein occlusion, ${ }^{6}$ branch retinal vein occlusion, ${ }^{7}$ uveitis, $^{8}$ pseudophakia, ${ }^{9}$ and telangiectasia. ${ }^{3}$ Indeed, some authors are even using IVT therapeutic agents for relatively rare disorders, such as central serous chorioretinopathy. $^{3}$

Patients' visual perceptions and experiences induced by frequently performed ophthalmic surgical procedures, such as cataract surgery (under topical ${ }^{10-15}$ and regional anesthesia $^{11-14,16,17}$ ), glaucoma filtration 
surgery, ${ }^{18}$ and vitreoretinal surgery, ${ }^{19-22}$ are well documented. Indeed, the beneficial effect of preoperative counseling with respect to known and anticipated visual experiences during phacoemulsification cataract surgery, under topical anesthesia, in terms of reducing patients' fear, has been described. ${ }^{23}$

However, to our knowledge, there has been no report of patients' subjective visual experiences induced by IVT injections. Awareness of patients' visual experiences in the immediate and early post-injection periods will enhance counseling in the preinjection setting, and may avert unjustified concern in the post-injection period on the part of patients undergoing IVT injections of therapeutic agents.

We have executed and report a questionnaire-based survey, designed to investigate and document visual perceptions induced by IVT injections of therapeutic agents.

\section{Patients and methods}

Patients undergoing an IVT injection of ranibizumab, pegaptanib sodium, or triamcinolone acetonide by a single surgeon (SB) between September 2009 and December 2009 were included in the survey. We recruited 82 patients (94 eyes), who underwent a total of 180 consecutive IVT injections and who, following each IVT injection, answered the immediate post-injection questionnaire. Only one injection episode per patient was selected for evaluation (the first one, in cases of serial injections) to avoid subject bias. Each patient was furnished with a detailed information leaflet regarding the risks and benefits of IVT administration of therapeutic agents, including blindness and loss of an eye, and provided written and informed consent for the proposed procedure. Of note, the leaflet did not allude to possible visual perceptions following the injection. Each patient provided informed consent before participating in the questionnaire-based survey. Ethics committee approval was secured from the Local Regional Ethics Committee and the research was conducted in accordance with the tenets of the Declaration of Helsinki.

The IVT injection was performed under direct non-microscopic visualization, under topical anesthesia (single-dose, preservative-free guttae proxymetacaine hydrochloride $0.5 \% \mathrm{w} / \mathrm{v}$ (Minims Proxymetacaine $0.5 \%$ Eye Drops, Chauvin, Kingston-upon-Thames, UK)) and using aseptic technique, in accordance with existing consensus guidelines. ${ }^{24-26}$ The pupil was pharmacologically dilated before the IVT injection with single-dose preservative-free guttae cyclopentolate hydrochloride 1\% w/v (Minims Cyclopentolate Hydrochloride) and single-dose preservative-free guttae tropicamide $1 \% \mathrm{w} / \mathrm{v}$ (Minims Tropicamide), to allow visualization of the central retinal artery (CRA) immediately after injection. Visualization of the CRA was achieved using a head-mounted indirect ophthalmoscope until such time, as the pulsatile or perfused status of the optic nerve head was confirmed. All patients were given information on signs and symptoms of endophthalmitis, and other clinically important symptoms, and instructed to present immediately should such symptoms occur. Of note, inadvertent injection of an air bubble was not observed in any of the cases.

An initial questionnaire was administered by the authors, in the immediate post-injection period, and only after confirmation of the pulsatile or perfused status of the CRA (Table 1). It contained three questions, all leading in nature. The first two questions offered 'yes' or 'no' forced-choice alternate options. The last question offered 'better', 'worse', or 'same' forced-choice alternate-options. The person administering the questionnaire covered the patient's other eye for the duration of completion of the questionnaire and asked $\mathrm{him} /$ her to keep the injected eye open while answering the questions.

All patients were discharged the same day and routine follow-up was scheduled for 2 weeks following the injection.

At the time of the 2-week follow-up, a second questionnaire was given to the patients (Table 1). The questionnaire was self-administered in the cases in which the patients' visual acuity (VA) allowed them to read it, and administered by the investigators in cases in which the patient's VA prevented him/her from reading the questionnaire. It contained six questions, all leading in nature, relating to visual experiences in the injected eye over the preceding 2 weeks. The first three questions were identical to the ones in the immediate post-injection questionnaire. Of the additional three questions, two questions offered 'yes' or 'no' forced-choice alternate options, and one question offered a visual analog scale (VAS) from 0 to 10 and asked the patients to grade any fear/distress/concern arising from the visual perceptions experienced since the IVT injection.

The mean follow-up was 15.2 days (SD: 2.8; range: 8-26). No intra- or post-operative complications occurred.

Statistical analysis was performed using the software package PASW Statistics 18.0 (IBM Corporations, Somers, NY, USA). The responses to questionnaire items in the immediate and 2-week recollection questionnaires were analyzed for the entire group and for the following injection subgroups: no previous injection vs previous injection(s) in the study eye; injections in study eyes with good VA (logarithm of minimal angle of resolution $[\log \mathrm{MAR}] \leq 0.3)$ vs moderate VA $(0.7<\log \mathrm{MAR}>0.3)$ vs 

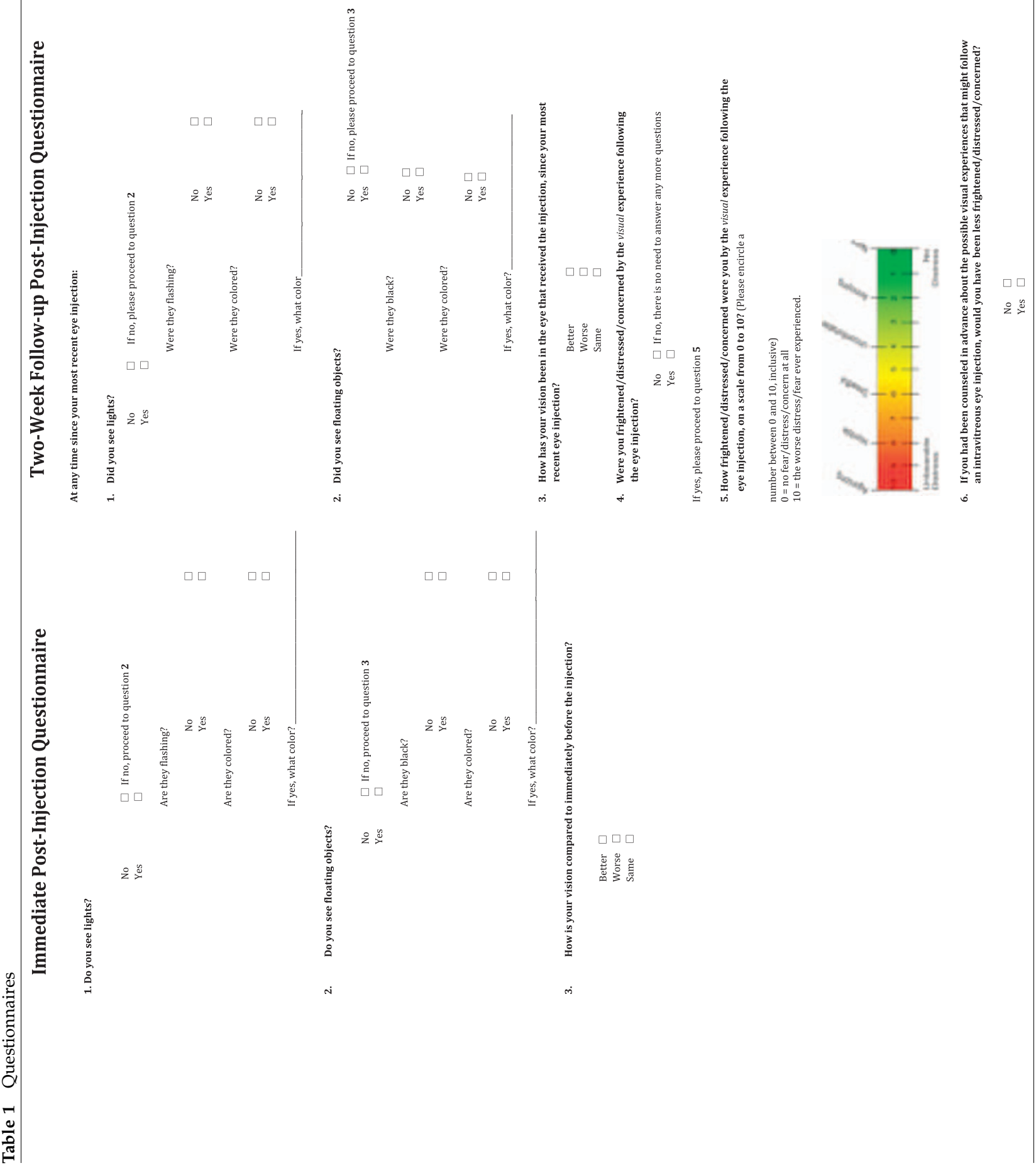
Table 2 Comparison of responses to the immediate post-injection and the 2-week recollection questionnaires

\begin{tabular}{|c|c|c|c|c|c|}
\hline \multicolumn{2}{|c|}{ Questions } & \multirow[b]{2}{*}{ Answer } & \multicolumn{2}{|c|}{ Answers } & \multirow[t]{2}{*}{ P-value } \\
\hline Immediately post-injection & At 2-week recollection & & $\begin{array}{l}\text { Immediately } \\
\text { post-injection }\end{array}$ & $\begin{array}{l}\text { At 2-week } \\
\text { recollection }\end{array}$ & \\
\hline Do you see lights? $(n=75)$ & Did you see lights? $(n=75)$ & Yes & $20(27 \%)$ & $11(15 \%)$ & 0.06 \\
\hline Flashing? $(n=20)$ & Flashing? $(n=27)$ & Yes & $11(55 \%)$ & $7(64 \%)$ & 0.56 \\
\hline Colored? $(n=20)$ & Coloured? $(n=27)$ & Yes & $13(65 \%)$ & $5(45 \%)$ & 0.56 \\
\hline \multirow[t]{4}{*}{ What colour? $(n=13)$} & What colour? $(n=14)$ & Blue/purple & 8 & 1 & \\
\hline & & Silver/white & 4 & 1 & \\
\hline & & Red & 1 & 1 & \\
\hline & & Yellow & 0 & 2 & \\
\hline Do you see floaters? $(n=75)$ & Did you see floaters? $(n=75)$ & Yes & $24(32 \%)$ & $48(64 \%)$ & 0.00 \\
\hline Black? $(n=24)$ & Black? $(n=48)$ & Yes & $14(58 \%)$ & $41(85 \%)$ & 0.21 \\
\hline Colored? $(n=24)$ & Colored? $(n=48)$ & Yes & $8(33 \%)$ & $7(15 \%)$ & 0.32 \\
\hline \multirow[t]{3}{*}{ What colour? $(n=8)$} & What colour? $(n=7)$ & Blue/purple & 4 & 3 & \\
\hline & & Silver/white & 2 & 4 & \\
\hline & & Red & 2 & 0 & \\
\hline \multirow[t]{3}{*}{$\begin{array}{l}\text { Vision now compared with } \\
\text { vision before injection }(n=75)\end{array}$} & $\begin{array}{l}\text { Vision now compared with } \\
\text { before injection }(n=75)\end{array}$ & Better & $7(10 \%)$ & $33(44 \%)$ & 0.00 \\
\hline & & Worse & $49(65 \%)$ & $4(5 \%)$ & \\
\hline & & Same & $19(25 \%)$ & $38(51 \%)$ & \\
\hline- & $\begin{array}{l}\text { Concern with visual } \\
\text { experience? }(n=75)\end{array}$ & Yes & - & $11(15 \%)$ & - \\
\hline- & $\begin{array}{l}\text { Would counseling have } \\
\text { reduced the concern? }(n=11)\end{array}$ & Yes & - & $7(64 \%)$ & - \\
\hline- & VAS score $(n=18)$ & - & - & $\begin{array}{r}\text { Mean }( \pm \text { SD }) \\
4.5( \pm 1.7)\end{array}$ & - \\
\hline
\end{tabular}

Abbreviations: SD, standard deviation; VAS, visual analog scale.

poor VA (logMAR $\geq 0.7)$; injections according to pharmacological agent (ranibizumab vs pegaptanib vs triamcinolone acetonide); injections in study eyes with $\mathrm{CNV}$ (of various causes) vs study eyes with $\mathrm{MO}$ (of various causes); and injections in phakic vs pseudophakic study eyes. Descriptive statistics were used to analyze the responses to each questionnaire item in the entire group. A series of Wilcoxon signed-rank tests were performed to compare responses immediately after injection and at the 2-week follow-up. A series of $\chi^{2}$ tests were performed to compare the responses to each questionnaire item in the predefined subgroups, and a one-way analysis of variance was performed to compare the VAS scores between subgroups. A $P$-value of less than 0.05 was considered statistically significant.

\section{Results}

After implementation of the exclusion criteria, data from 75 patients (75 eyes), who underwent one IVT injection but completed both questionnaires, were evaluated. The mean $( \pm S D)$ age was $74.3( \pm 9.7)$ years. The male/female ratio was $25: 50$, and the right eye: left eye ratio was $33: 42$. In total, 45 patients $(60 \%)$ had a history of IVT injection(s) before recruitment in the study.

\section{Analysis of entire group}

The responses in the entire group, to each questionnaire item, immediately after an IVT injection $(n=75)$, and at the 2-week follow-up ( $n=75)$, are given in Table 2. Lights were observed immediately following $27 \%$ (20/75) of IVT injections, and were flashing $(11 / 20 ; 55 \%)$ and colored $(13 / 20 ; 65 \%)$ in over half of these cases. The answers at the time of completion of the 2-week recollection questionnaire, with respect to perceived lights, were not statistically different from those reported in the immediate post-injection period (Wilcoxon signedrank tests: $P>0.05$ for all).

Floaters were observed immediately after 24/75 (32\%) IVT injections, and were black in $14 / 24(58 \%)$ of these cases and colored in 8/24 (33\%) of these cases, with blue and purple being the predominant colors reported. At the time of completion of the 2-week recollection questionnaire, there was a statistically significant increase in the number of IVT injections that had caused floaters, with floaters reported after $64 \%$ of IVT injections, compared with only $32 \%$ of IVT injections in the immediate post-injection period (Wilcoxon signed-rank tests: $P=0.00$ ). The rest of the answers, at the time of completion of the 2-week recollection questionnaire, 
with respect to floaters, were not statistically different from those reported in the immediate post-injection period (Wilcoxon signed-rank tests: $P>0.05$ for all).

At the time of completion of the 2-week recollection questionnaire, visual perceptions experienced following $15 \%(n=11)$ of IVT injections had given cause for concern/distress to those undergoing the injection (mean VAS score ( \pm SD): $4.5( \pm 1.7))$, and in $64 \%$ of these cases $(n=7)$ it was believed by the patient that preoperative counseling would have alleviated the expressed concern. Finally, the reported quality of vision changed significantly between the immediate post-injection period and the time of completion of the 2-week recollection questionnaire, with better, similar, and worse vision reported following 44,51 , and $5 \%$ of injections at the 2-week follow-up, respectively (compared with following 10, 25, and $65 \%$ of IVT injections, respectively, immediately after injection; Wilcoxon signed-rank tests: $P=0.00)$.

\section{Subgroup analysis}

Subgroup analysis of responses to each questionnaire item was performed for responses given immediately after an IVT injection (Table 3 ) and for responses given at the time of completion of the 2-week recollection questionnaire (Table 4). Both immediately after an IVT injection and at the time of completion of the 2-week recollection questionnaire, floaters were more likely to be reported in the suspension subgroup (triamcinolone acetonide; $4 / 6 ; 67 \%$ ) than in the clear-agent subgroup (ranibizumab or pegaptanib sodium; 20/69; 29\%; $\chi^{2}$-test: $P=0.06$ ), but the difference was statistically borderline. Similarly, floaters were more likely to be reported by patients receiving an injection for the treatment of $\mathrm{MO}(7 / 13 ; 54 \%)$ than by patients receiving an injection for the treatment of choroidal neovascular membrane $(17 / 62 ; 27 \%)\left(\chi^{2}\right.$-test: $\left.P=0.06\right)$, but again, the difference was statistically borderline. Subgroups did not differ in any other respect of visual perception, either immediately following the IVT injection or at the time of completion of the 2-week recollection questionnaire $\left(\chi^{2}\right.$-test; $P>0.05$ for all remaining findings).

\section{Discussion}

We executed and report a questionnaire-based survey, designed to investigate and document subjective visual perceptions induced by IVT injections of therapeutic agents. We believe that awareness of patients' visual experiences in the immediate and early post-injection periods is important for the purposes of preoperative counseling and informed consent, and to satisfactorily

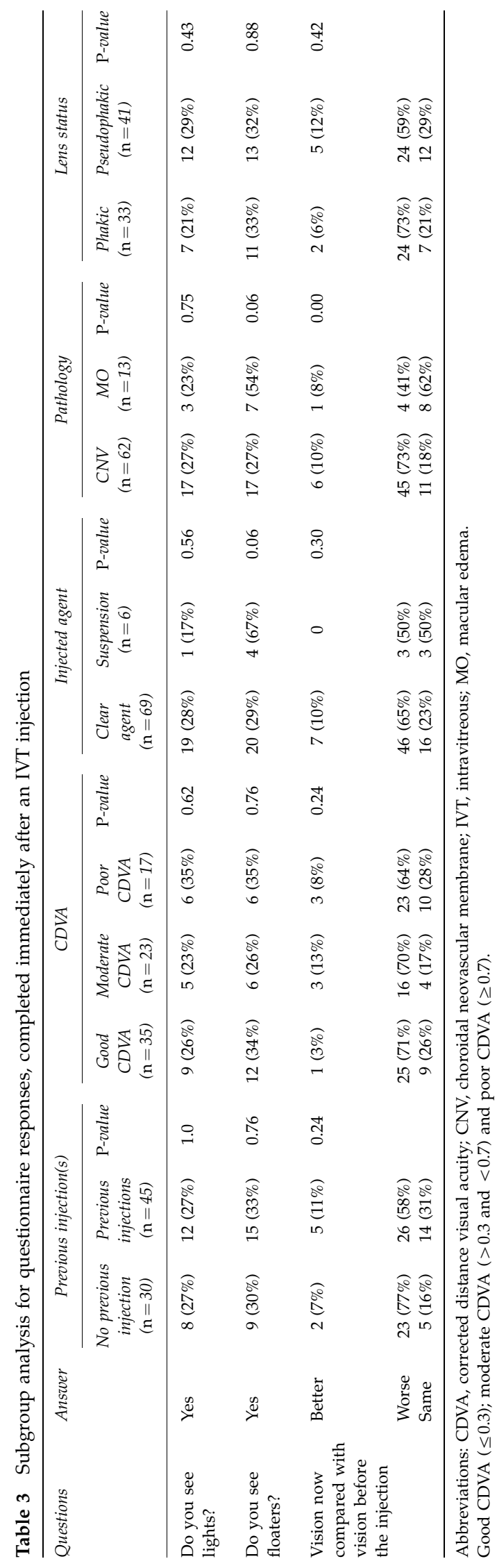


address patients' telephone queries in the early post-injection period.

To the authors' knowledge, there are no published reports of visual perceptions following IVT injections of therapeutic agents, although a number of studies have been performed on the visual phenomena experienced by patients during phacoemulsification cataract surgery. ${ }^{10-17}$

We report that flashing lights and floaters are commonly experienced by patients immediately following an IVT injection, and that flashes are experienced by almost two-thirds of patients within the first 2 weeks following the procedure. Preinjection VA, clinical indication for the IVT injection, and the nature of the pharmacological agent injected did not influence the visual experiences of patients, although the authors concede that subgroups analysis was limited because of its small size. Of note, visual experiences following $15 \%$ of IVT injections gave cause for concern on the part of patients.

It has been postulated that the visual phenomena experienced by patients during phacoemulsification cataract surgery might represent a combination of colored entoptic phenomena, light-induced visual sensations, and afterimages, ${ }^{27}$ and it is likely that a number of well-described entoptic phenomena ${ }^{28}$ might be similarly responsible for the visual experiences described by patients after an IVT injection. First, for example, droplets and threads of guttae fusidic acid, a substance, which because of its viscous nature, causes local variation in the refractive index of the cornea, might cause streaks and spots of light, ${ }^{28}$ as reported by some patients in this study. Of note, the authors do concede that the presence of viscous guttae fusidic acid on the ocular surface immediately after injection (which was part of the post-injection protocol) may have contributed to the blurring of vision reported by some patients in the immediate post-injection period. Moreover, a transient elevation of intraocular pressure immediately after injection (attested to by the pulsatile nature of the CRA of the injected eye) could cause corneal epithelial edema and consequential halos of light. ${ }^{28}$ Further, vitreous opacities are known to cast umbral shadows on the retina that give rise to positive scotomas, and are reported by patients as floaters, shadows, or chains of bright spots like a string of pearls, ${ }^{28}$ and this is a well-described effect of an IVT injection of triamcinolone acetonide (which is opaque). However, it is possible that ranibizumab and pegaptanib sodium, even though transparent, would cause local variation in the refractive index of the vitreous, thereby giving rise to similar visual experiences. Also, direct mechanical stimulation of the retina is known to cause a luminous sensation 
called a phosphene, ${ }^{28}$ and it is possible that the mechanical pressure applied locally on the sclera at the point of injection, firstly by the calipers and then by the needle, might induce such phosphenes following an IVT injection. Finally, the light source of the head-mounted indirect ophthalmoscope, used to visualize the CRA at the end of the procedure, could give rise to Purkinje's figures (a percept of the retinal vascular tree, reported by surveyed patients as shadows or cobwebs), ${ }^{28}$ if the direction of the incident light changed quickly during the examination.

Awareness of the nature and duration of visual phenomena, induced by IVT injections, should represent an important component of patient preinjection counseling, and will facilitate the healthcare worker dealing with patients' queries (by telephone or otherwise) regarding their IVT injection in the preinjection and in the post-injection period. Furthermore, and in an era of falling thresholds for surgical intervention (and associated high expectations of patients), inadequate informed consent remains the most important factor in cases of medico-legal litigation. ${ }^{29-31}$ We believe that knowledge of the nature and duration of visual perceptions attributable to IVT injections, reported here for the first time, will furnish the practicing ophthalmologist with the wherewithal to strengthen the process of informed consent in the preinjection setting.

In conclusion, lights and floaters are frequent visual perceptions following an IVT injection of therapeutic agents. The majority of participants reported that the concern arising from these visual experiences would have been alleviated if they had been counseled before the injection. Hence, the data from this study will inform professional practice and patients' expectations following the procedure alleviate concerns in the immediate and early post-injection periods, and enhance the process of informed consent.

\section{Summary}

What was known before

- Floaters are frequent visual perceptions following IVT injections of triamcinolone acetonide.

What this study adds

- Lights and floaters are frequent visual perceptions following IVT injections of ranibizumab, pegaptanib sodium, and triamcinolone acetonide. They can give cause for concern that could be alleviated with pre-injection counseling.

\section{Conflict of interest}

The authors declare no conflict of interest.

\section{References}

1 Rosenfeld PJ, Brown DM, Heier JS, Boyer DS, Kaiser PK, Chung CY et al. Ranibizumab for neovascular age-related macular degeneration. N Engl J Med 2006; 355(14): 1419-1431.

2 Brown DM, Michels M, Kaiser PK, Heier JS, Sy JP, Ianchulev T. Ranibizumab versus verteporfin photodynamic therapy for neovascular age-related macular degeneration: two-year results of the ANCHOR study. Ophthalmology 2009; 116(1): 57-65.e5.

3 Lantry LE. Ranibizumab, a mAb against VEGF-A for the potential treatment of age-related macular degeneration and other ocular complications. Curr Opin Mol Ther 2007; 9(6): 592-602.

4 Querques G, Bux AV, Martinelli D, Iaculli C, Noci ND. Intravitreal pegaptanib sodium (Macugen) for diabetic macular oedema. Acta Ophthalmol 2009; 87(6): 623-630.

5 Yilmaz T, Weaver CD, Gallagher MJ, Cordero-Coma M, Cervantes-Castaneda RA, Klisovic D et al. Intravitreal triamcinolone acetonide injection for treatment of refractory diabetic macular edema: a systematic review. Ophthalmology 2009; 116(5): 902-911; quiz 912-913.

6 Gewaily D, Greenberg PB. Intravitreal steroids versus observation for macular edema secondary to central retinal vein occlusion. Cochrane Database Syst Rev 2009; (1): CD007324.

7 Wroblewski JJ, Wells III JA, Gonzales CR. Pegaptanib sodium for macular edema secondary to branch retinal vein occlusion. Am J Ophthalmol 2010; 149(1): 147-154.

8 Cervantes-Castaneda RA, Giuliari GP, Gallagher MJ, Yilmaz T, MacDonell RE, Quinones $\mathrm{K}$ et al. Intravitreal bevacizumab in refractory uveitic macular edema: one-year follow-up. Eur J Ophthalmol 2009; 19(4): 622-629.

9 Cervera E, Diaz-Llopis M, Udaondo P, Garcia-Delpech S. Intravitreal pegaptanib sodium for refractory pseudophakic macular oedema. Eye 2008; 22(9): 1180-1182.

10 Ang CL, Au Eong KG, Lee SS, Chan SP, Tan CS. Patients' expectation and experience of visual sensations during phacoemulsification under topical anaesthesia. Eye 2007; 21(9): 1162-1167.

11 Tranos PG, Wickremasinghe SS, Sinclair N, Foster PJ, Asaria R, Harris ML et al. Visual perception during phacoemulsification cataract surgery under topical and regional anaesthesia. Acta Ophthalmol Scand 2003; 81(2): 118-122.

12 Rengaraj V, Radhakrishnan M, Au Eong KG, Saw SM, Srinivasan A, Mathew J et al. Visual experience during phacoemulsification under topical versus retrobulbar anesthesia: results of a prospective, randomized, controlled trial. Am J Ophthalmol 2004; 138(5): 782-787.

13 Chung CF, Lai JS, Lam DS. Visual sensation during phacoemulsification and intraocular lens implantation using topical and regional anesthesia. J Cataract Refract Surg 2004; 30(2): 444-448.

14 Tan CS, Au Eong KG, Kumar CM. Visual experiences during cataract surgery: what anaesthesia providers should know. Eur J Anaesthesiol 2005; 22(6): 413-419.

15 Yaylali V, Yildirim C, Tatlipinar S, Demirlenk I, Arik S, Ozden S. Subjective visual experience and pain level during phacoemulsification and intraocular lens implantation under topical anesthesia. Ophthalmologica 2003; 217(6): 413-416. 
16 Prasad N, Kumar CM, Patil BB, Dowd TC. Subjective visual experience during phacoemulsification cataract surgery under sub-Tenon's block. Eye 2003; 17(3): 407-409.

17 Wickremasinghe SS, Tranos PG, Sinclair N, Andreou PS, Harris ML, Little BC. Visual perception during phacoemulsification cataract surgery under subtenons anaesthesia. Eye 2003; 17(4): 501-505.

18 Riad W, Tan CS, Kumar CM, Au Eong KG. What can patients see during glaucoma filtration surgery under peribulbar anesthesia? J Glaucoma 2006; 15(5): 462-465.

19 Vohra SB, Anya C, Farooq T, Murray PI. Subjective visual perceptions during vitreoretinal surgery under local anaesthesia. Eye 2009; 23(9): 1831-1835.

20 Tan CS, Mahmood U, O'Brien PD, Beatty S, Kwok AK, Lee VY et al. Visual experiences during vitreous surgery under regional anesthesia: a multicenter study. $A m \mathrm{~J}$ Ophthalmol 2005; 140(6): 971-975.

21 Sugisaka E, Shinoda K, Sano RY, Ishida S, Imamura Y, Ozawa $Y$ et al. Mechanism of visual sensations experienced during pars plana vitrectomy under retrobulbar anesthesia. Ophthalmologica 2009; 224(2): 103-108.

22 Kawaguchi N, Inoue M, Sugisaka E, Shinoda K, Tsubota K. Subjective visual sensation during vitrectomy under retrobulbar anesthesia. Am J Ophthalmol 2006; 141(2): 407-409.

23 Voon LW, Au Eong KG, Saw SM, Verma D, Laude A. Effect of preoperative counseling on patient fear from the visual experience during phacoemulsification under topical anesthesia: multicenter randomized clinical trial. J Cataract Refract Surg 2005; 31(10): 1966-1969.

24 Aiello LP, Brucker AJ, Chang S, Cunningham Jr ET, D'Amico DJ, Flynn Jr HW et al. Evolving guidelines for intravitreous injections. Retina 2004; 24(5 Suppl): S3-S19.

25 Bylsma G, Guymer R, Qureshi S, Gin T, Allen P, Madhok P et al. Intravitreous injections. Clin Experiment Ophthalmol 2006; 34(4): 388-390.

26 Rodrigues EB, Meyer CH, Grumann Jr A, Shiroma H, Aguni JS, Farah ME. Tunneled scleral incision to prevent vitreal reflux after intravitreal injection. Am J Ophthalmol 2007; 143(6): 1035-1037.

27 Sumich PM, Francis IC, Kappagoda MB, Alexander SL. Artist's impression of endocapsular phacoemulsification surgery. J Cataract Refract Surg 1998; 24(11): 1525-1528.

28 Kaufman PL, Alm A. Adler's Physiology of the Eye: Clinical Application, 10th edn. Mosby: St Louis, MO, 2003.

29 Reynard J, Marsh H. Unusual and not so unusual ways of ending up in court: how to avoid litigation. BJU Int 2009; 104(5): 586-589.

30 Brosnam T, Perry M. 'Informed' consent in adult patients: can we achieve a gold standard? Br J Oral Maxillofac Surg 2009; 47(3): 186-190.

31 Olumide Olufowote J. A structurational analysis of informed consent to treatment: (re)productions of contradictory sociohistorical structures in practitioners' interpretive schemes. Qual Health Res 2009; 19(6): 802-814. 